\title{
Design of an Implantable Intracortical Microelectrode System for Brain-Machine Interfaces
}

\author{
Erin Patrick ${ }^{1}$, Viswanath Sankar ${ }^{1}$, William Rowe ${ }^{1}$, Justin C. Sanchez ${ }^{2}$, and Toshikazu Nishida ${ }^{1}$ \\ ${ }^{1}$ Department of Electrical and Computer Engineering, and ${ }^{2}$ Department of Pediatrics, Division of Neurology \\ University of Florida, Gainesville Florida, USA
}

\begin{abstract}
The long-term goal in the design of Brain-Machine Interfaces is to restore communication and control to unrestrained individuals. One of the great challenges in this effort is to develop implantable systems that are capable of processing the activity of large ensembles of cortical neurons. Here, we present the design, fabrication, and testing of a flexible microelectrode array that can be hybrid-packaged with custom electronics in a fully implantable form factor. The design specifications and process flow for incorporating flip-chip bonding of an amplifier die are discussed.
\end{abstract}

Keywords-component; microelectrode array, Brain-Machine Interface, implantable, neural recording

\section{INTRODUCTION}

The challenge of realizing wireless implantable neural recording systems for Brain-Machine Interfaces has been undertaken by several institutions $[1,2,3]$. Several designs have been proposed for hybrid integration with electronics. The Utah system integrates interface electronics via flip-chip bonding techniques to the top of a Si micromachined $100 \times 100$ electrode array implanted into the cortex [2]. The Michigan system uses a wafer level fabricated flexible cable to rout the signals from the implanted $\mathrm{Si}$ micromachind electrodes to a platform containing hybrid-packaged interface electronics located some distance away on the top of the skull $[1,4]$. Here we take another approach with our efforts at the University of Florida in the Florida Wireless Integrated Recording Electrode (FWIRE) project, which capitalize on the integration of two key technologies: a flexible microelectrode array substrate to couple electrodes with electronics [7] and a novel pulsed-based data representation scheme, which minimizes transmission bandwidth and allows spike sorting after transmission $[5,6]$. To achieve our goal of a fully implantable system, a custom microelectrode for application specific integrated circuit (ASIC) incorporation is underway. This paper defines the design and progress of a flexible substrate microelectrode array with a hybrid packaged integrated circuit amplifier for in vivo studies.

\section{DESIGN}

FWIRE design considerations include:

1. a platform with electronics residing on the skull reducing heat transfer to brain tissue,

2. a seamless connection between implanted electrodes and platform for electronics,
3. a grounding scheme that doubles as an anchoring mechanism,

and

4. hybrid-packaging techniques that allow attachment of multiple integrated circuits.

The guiding principle is the use of a relatively simple process flow utilizing tungsten microwires that have been used in vivo previously with high neuronal yield in a robust and customizable manufacturing process [7]. The entire package is designed to be implantable using stereotaxic placement.

\section{A. System Layout}

To prototype our neural recording system with the constraints listed above, we will implement it in a rodent animal model. The system consists of a) electrodes that can either be inline or offset from the substrate, b) patterned wire traces in cable form connecting the electrode to the integrated electronics, c) an integrated amplifier, and d) an integrated ground bolt as shown in fig.1. Until the wireless component of this design is completed, we offer access to the amplifier via a standard Omnetics 18 pin connector. This will eventually be replaced in the final design.

The design space is a Sprague-Dawley rat as depicted in fig 1 . The electrodes must be implanted into a craniotomy $(+1 \mathrm{~mm}$ anterior to bregma, $2.5 \mathrm{~mm}$ lateral) at the site corresponding to the forelimb region of the motor cortex [8]. The layout of our design was changed from [7] such that the device can be implanted inline with the central axis of the rat skull as shown in fig. 1. The electrode sites were shifted left of the midline of the device for implantation in the appropriate area of the motor cortex. A slot for a second screw is also added in this design, allowing less critical placement by the surgeon. Also a perpendicularly mounted Omnetics connector is used in this design scheme.

This work was supported by a NIH NINDS grant (\#NS053561). 


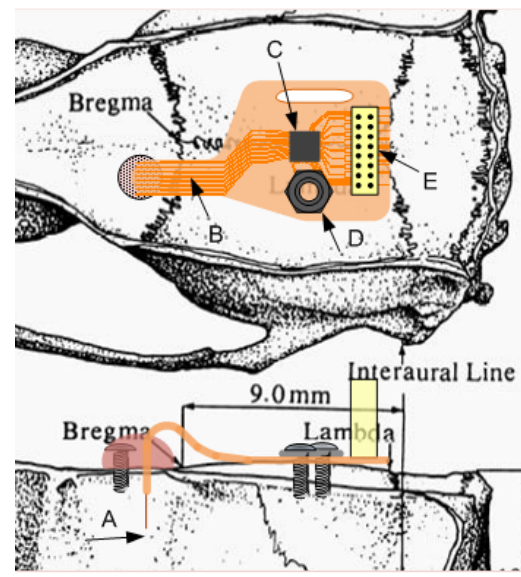

A - Tungsten microwire electrodes

B - Polyimide cable

C - Amplifier IC

D - Ground bolt

E - Omnetics connector

Figure 1. In vivo placement of microelectrode array on rodent skull. Inset shows full view.

\section{B. Process flow}

A process flow similar to that of our earlier generation electrodes is followed [7,9]. All the steps are carried out on a 4" Si wafer with a thin aluminum film acting as the sacrificial layer. Patterned gold wirings $(0.1 \mu \mathrm{m}$ thick $)$ are sandwiched between two layers of polyimide that provide an insulating platform for the electrode. The top polyimide layer is selectively etched to release the vias for the attachment of the amplifier chip and to define the device outline. $50 \mu \mathrm{m}$ thick tungsten wires are manually attached to the gold traces and electrically connected with a conductive epoxy. The devices are released from the $\mathrm{Si}$ wafer by anodic etching of $\mathrm{Al}$ sacrificial layer, and the electrodes are hermetically sealed and secured with a silicone elastomer (PDMS). The two possible methods of filling the bond pad vias and other critical steps of the fabrication process are shown in fig. 2. As seen from the figure, the attachment of the amplifier IC is the final step of this process flow.

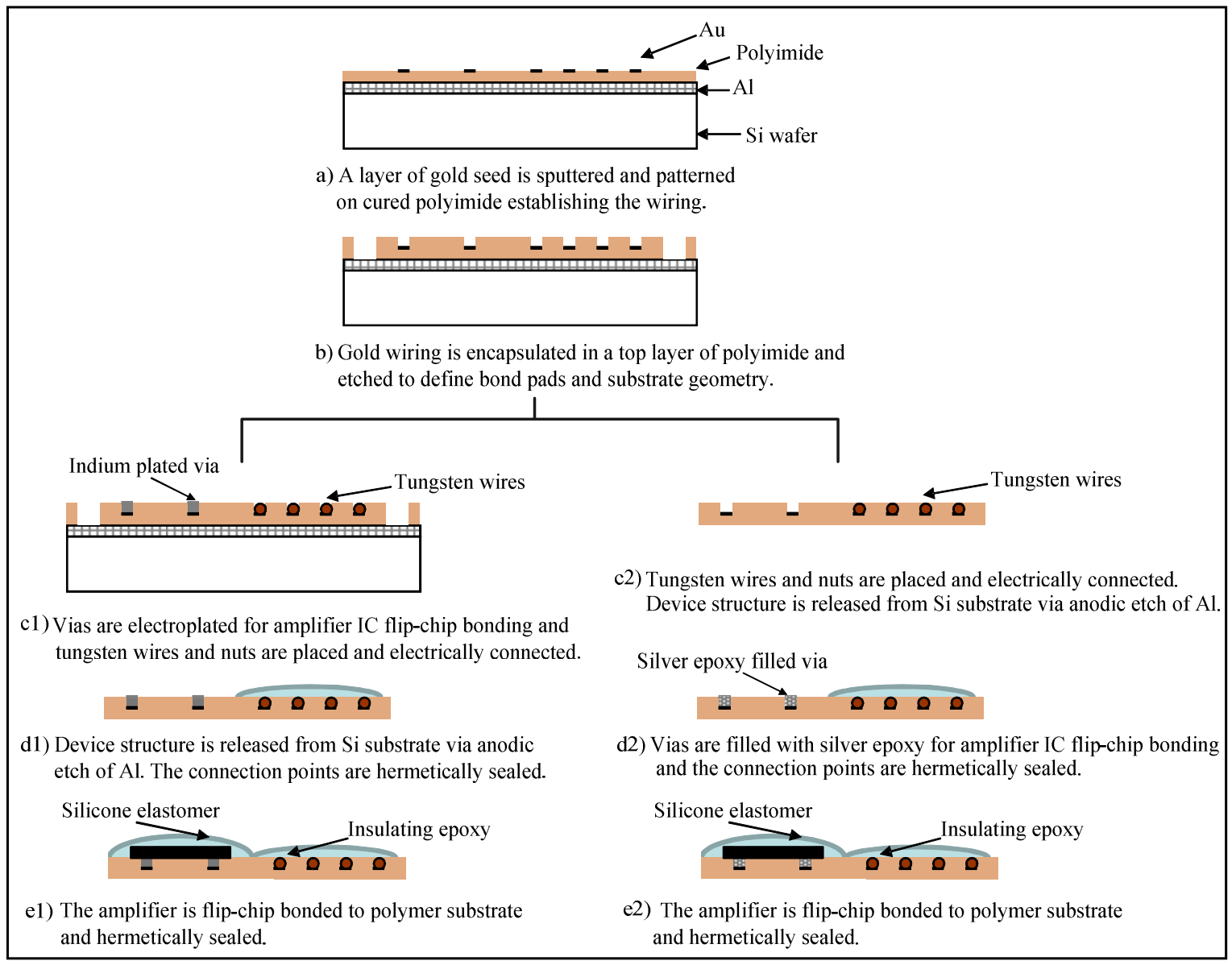

Figure 2. Process flow showing two avenues for establishing electrical contact between the amplifier IC and the electrode substrate. 


\section{Hybrid packaging of amplifier}

We are investigating two methods for the via fill for subsequent flip-chip bonding of the amplifier die: electroplated indium and micro-dispensed silver epoxy. Electrodeposition of indium bumps for flip-chip attachment of ICs has been practiced and proved successful [10]. Similarly, the use of a conductive silver epoxy as an electrical interface for flip-chip integration has been demonstrated [3]. The contact bond pads on the substrate have dimensions of $150 \mu \mathrm{m} \times 150 \mu \mathrm{m}$ to accommodate the chip bond pads with dimensions of $130 \mu \mathrm{m} \times$ $130 \mu \mathrm{m}$. The distance between two adjacent contact pads is 120 $\mu \mathrm{m}$.

\section{1) Electrodeposting In vias}

The electrodeposition process is carried out at room temperature with In as the anode, the device as the cathode and indium sulfamate bath (Indium Corp. of America) as the electrolyte. To establish a uniform foundation of In seed layer on the gold contact pads, we followed the "Strike" or "flash" plating scheme. In the strike plating scheme, a very high current density of $440 \mathrm{~mA} / \mathrm{cm}^{2}$ is applied for 5-6 sec. The strike method of plating In proved to reduce the surface roughness of the plated metal significantly. Further, to ensure a planar deposition in the via, we adhered to the "pulse" or the "periodic reverse" plating technique for the actual plating process. In the pulse plating method, the current is toggled between forward and reverse directions in a square wave cycle. The forward plating current is $20 \mathrm{~mA} / \mathrm{cm}^{2}$. The vias are filled with In to the height of the polyimide thickness plus $10 \mu \mathrm{m}$. Fig. 3 shows a picture of the electroplated indium via fill.

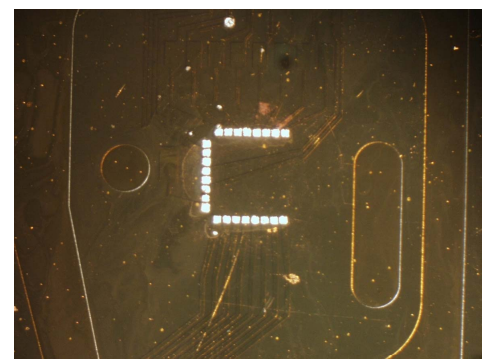

Figure 3. Electroplated indium via fill.

\section{2) Micro-dispensed Ag epoxy via fill}

The second approach to fill the via is micro-dispensing of silver epoxy. The gold contact pads are filled with conductive silver epoxy through a direct-write machine (nScrypt inc.) that is capable of dispensing micro droplets of silver epoxy into the vias. Fig. 4 shows an image of a trial run of the direct-write process. The droplets are $150 \mu \mathrm{m}$ in diameter and have a pitch of $105 \mu \mathrm{m}$.

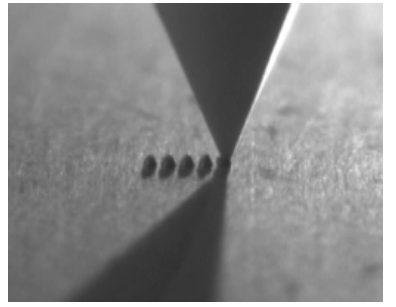

Figure 4. Dispensing of silver epoxy for via fill.

\section{3) Flip-chip bonding}

After the vias are filled with conductive materials, the amplifier chip is finally attached to the substrate by a flip-chip bonding process. To ensure an effective electrical contact to the pads of the amplifier die, gold stud bumps of 50-70 $\mu \mathrm{m}$ diameter are placed on the die bond pads by a wire bonding process. Fig. 5a shows the image of the chip with stud bumps. The flip-chip bonding process is performed on a flip-chip placement system (Model 850, Semiconductor Equipment Corp.) An in-house heating system is used to apply heat to the substrate during bonding with the indium via and after bonding with the sliver epoxy via to melt the In solder bumps and cure the silver epoxy. Fig. 5b shows the conceptual picture of the process. To establish a secure mechanical connection and hermetic seal of the chip with the polyimide substrate, the device is coated with a low moisture intake underfill epoxy (Epo-tek 302-3M).

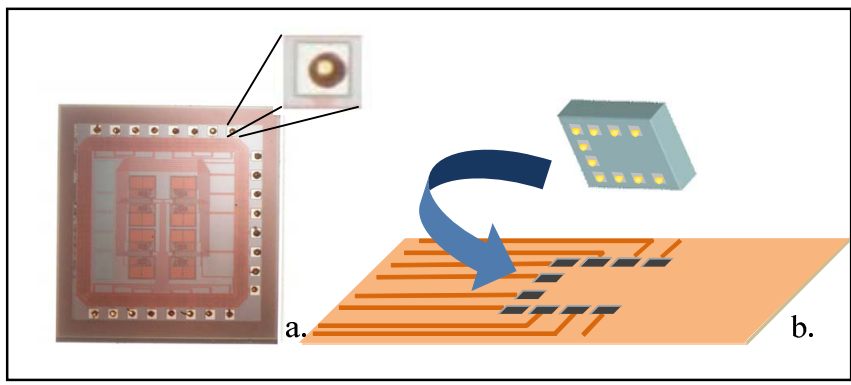

Figure 5. a) Amplifier die showing stud bumps on bond pads. b) Illustration of flip-chip bonding of amplifier die.

\section{PRELIMINARY Results AND DisCUSSION}

As stated above, two different approaches for creating the electrical contact bumps for flip-chip bonding of the amplifier are being investigated. The In electroplated bond pads were tested for flip-chip attachment of the amplifier on a prototype substrate without vias. $10 \mu \mathrm{m}$ thick In was electroplated onto a flat polyimide substrate. The amplifier IC was attached by the aforementioned flip-chip process; however, no heating was applied to reflow the In. Experiments conducted on this test bed proved that mechanical connection of the amplifier IC to the indium bondpads of the polyimide substrate was sufficient for electrical contact. To test this, a $100 \mu \mathrm{V}$ peak sine wave at $1 \mathrm{kHz}$ sourced by a spectrum analyzer (SRS780) was given as an input to the flip-chip bonded amplifier and a $9 \mathrm{mV}$ peak 
output was measured by the spectrum analyzer. This gain of 90 was consistent with the specifications of the amplifier.

One issue with the indium via fill methods is poor yield in the number of functional indium bondpads after all planar processing techniques are completed. Poor adhesion to the substrate occasionally causes the indium pads to release from the substrate during a necessary cleaning step after the final reactive ion etch of the polyimide. Thus, micro-dispensing silver epoxy is more attractive since this step will come last in the process flow prior to flip-chip bonding.

\section{SUMMARY}

Specific design considerations for the FWIRE project include: a subcutaneous platform housing the interface electronics placed away from the cortex on top of the skull, a mechanically reliable and flexible interface between the implanted electrodes and the electronics platform, and an anchoring scheme that reduces overall implant dimensions. In this paper, we show the design and preliminary results based on these considerations. Micromachining techniques are used to fabricate a polyimide substrate that connects tungsten mircowire electrodes to electronics. We show preliminary results of successful flip-chip bonding of a custom amplifier IC using indium bond pads and underfill insulating epoxy.

Future work involves bench-top testing, which includes verification of system specifications and in vivo validation of the hybrid-packaged microelectrode system.

\section{ACKNOLWEDGEMENTS}

We would like to acknowledge Dr. Eric Wachsman and Bryan Blackburn from the Materials Science Department in the University of Florida for the use of the nScript direct write machine and help with the silver epoxy via fill process.

\section{REFERENCES}

[1] K.D. Wise, A.M. Sodagar, Y. Yao, M. N. Gulari, G.E. Perlin, and K. Najafi, "Microelectrodes, microelectronics, and implantable neural microsystems", Proc. Of IEEE, Vol. 96, Iss. 7, pp. 1184-1202, July 2007.

[2] S. Kim, R. Bhandari, M. Klein, S. Negi, L. Reith, P. Tathireddy, M. Toepper, H. Oppermann, and F. Solzbacher,. "Integrated wireless neural interface based on the Utah electrode array." Biomedical Microdevices, Dec 2008.

[3] Y. Song, W.R. Patterson, C.W. Bull, D.A. Borton, Y. Li, A.V. Nurmikko, J. D. Simeral, and J. P. Donoghue, "A brain implantable microsystem with hybrid RF/IR telemetry for advanced neuroengineering applications", Proc. $29^{\text {th }}$ Ann. Int. Conf. IEEE EMBS, pp. 445-448, August 2007.

[4] A.M. Sodagar, G.E. Perlin, Y. Yao, K.D. Wise, and K. Najafi, "An implantable microsystem for wireless multi-channel cortical recording", Transducers 2007, pp. 69-72, June 2007.

[5] J.G. Harris, J.C. Principe, J.C. Sanchez, D. Chen, and C. She, "Pulsebased signal compression for implanted neural recording systems", IEEE ISCAS, pp. 344-347, May 2008.
[6] R. Bashirullah, J. G. Harris, J.C. Sanchez, T. Nishida, and J. C. Principe, "Florida Wireless Implantable Recording Electrodes (FWIRE) for brain machine interfaces", IEEE ISCAS, pp. 2084-2087, May 2007.

[7] E. Patrick, V. Sankar, W. Rowe, S.F. Yen, J.C. Sanchez, and T. Nishida, "Flexible polymer substrate and tungsten microelectrode array for an implantable neural recording system", Proc. $30^{\text {th }}$ Ann. Int. Conf.IEEE EMBS, pp. 3158-3161, 2008.

[8] G. Paxinos, "The rat brain in stereotaxic coordinates", Sydney, Academic Press. 1997.

[9] E. Patrick, M. Ordonez, N. Alba, J.C. Sanchez, and T. Nishida, "Design and fabrication of a flexible substrate microelectrode array for brain machine interfaces," Proc. $28^{\text {th }}$ Ann. Int. Conf.IEEE EMBS, pp. 29662969, 2006.

[10] Y.Tian, C. Liu, D. Hutt, and B. Stevens, "Electrodeposition of indium for bump bonding", $58^{\text {th }}$ IEEE Electronic Components and Technology Conference, pp. 2096-2010, May 2008. 